PROCEEDINGS OF THE

AMERICAN MATHEMATICAL SOCIETY

Volume 133, Number 7, Pages 1941-1947

S 0002-9939(05)07843-3

Article electronically published on January 31, 2005

\title{
THERE IS NO BOGOMOLOV TYPE RESTRICTION THEOREM FOR STRONG SEMISTABILITY IN POSITIVE CHARACTERISTIC
}

\author{
HOLGER BRENNER
}

(Communicated by Michael Stillman)

\begin{abstract}
We give an example of a strongly semistable vector bundle of rank two on the projective plane such that there exist smooth curves of arbitrary high degree with the property that the restriction of the bundle to the curve is not strongly semistable anymore. This shows that a Bogomolov type restriction theorem does not hold for strong semistability in positive characteristic.
\end{abstract}

\section{INTRODUCTION}

A locally free sheaf $\mathcal{S}$ on a smooth projective variety $X$ is called semistable if for every coherent torsion-free subsheaf $\mathcal{T} \subseteq \mathcal{S}$ the inequality $\mu(\mathcal{T}) \leq \mu(\mathcal{S})$ holds, where the slope is defined by $\mu(\mathcal{T})=\operatorname{deg}(\mathcal{T}) / \operatorname{rk}(\mathcal{T})$ and the degree is defined with respect to a fixed ample divisor $H$ on $X$ by $\operatorname{deg}(\mathcal{T})=\operatorname{deg}(\operatorname{det}(\mathcal{T}))=\operatorname{det}(\mathcal{T}) \cdot H^{\operatorname{dim} X-1}$. On a curve, this notion is independent of an ample divisor and on a projective space we always take $H=\mathcal{O}(1)$.

A natural question is whether the restriction of a semistable vector bundle to a hypersurface is again semistable. One motivation for this is the attempt to reduce boundedness questions to lower-dimensional varieties. Let us recall briefly what is known in the easiest case, for bundles on the projective plane and their restrictions to curves (see [9 Chapter 7]). One kind of result is that the restriction of a semistable bundle to a generic curve of sufficiently high degree is again semistable. The theorem of Flenner (5]) gives an explicit bound for the degree of the curve in characteristic zero; the theorem of Mehta and Ramanathan (12]) holds in arbitrary characteristic, but without a degree bound.

The theorem of Bogomolov ([1]) shows in characteristic zero that the restriction of a stable bundle is again stable for every smooth curve of sufficiently high degree, not only for the generic curve. A. Langer has recently shown that such a restriction theorem holds also in positive characteristic; see [11, Theorem 5.2].

A well known phenomenon in positive characteristic is that the pull-back of a semistable sheaf under the Frobenius morphism is in general no longer semistable. A bundle is called strongly semistable if every Frobenius pull-back of it is again semistable. A semistable bundle on the projective plane is also strongly semistable (the same is true for elliptic curves). What is known about restriction of strong

Received by the editors February 10, 2004 and, in revised form, March 20, 2004.

2000 Mathematics Subject Classification. Primary 14J60, 14H60, 13A35.

(C) 2005 American Mathematical Society Reverts to public domain 28 years from publication 
semistability? A. Langer has shown in positive characteristic that under the condition that the discriminant of the bundle is 0 , then its restriction to a very general curve is strongly semistable [11, Theorem 3.1 and Remark 3.10.2].

The general question how strong semistability behaves under restriction is open. In this paper we show by an example that we cannot hope for the best possible result, that is, a result à la Bogomolov does not hold: there is no restriction theorem for strong semistability which holds for all smooth curves of sufficiently high degree.

Our example is motivated by the theory of tight closure and is quite easy to write down; it is the syzygy bundle $\operatorname{Syz}\left(X^{a}, Y^{a}, Z^{a}\right)$ on the projective plane (which is the cotangent bundle for $a=1$ ). We show that for every $d_{0}$ there exists a $d \geq d_{0}$ (depending on the characteristic $p$ ) such that the restriction to the smooth Fermat curve $X^{d}+Y^{d}+Z^{d}=0$ is not strongly semistable.

The content of this paper is as follows: We describe the relevant properties of the example in section 1 In section 2 we make several remarks concerning further implications of the example, in particular with respect to tight closure. We show that $(Y X Z)^{b} \in\left(X^{2 b}, Y^{2 b}, Z^{2 b}\right)^{*}$ (which is always true in zero characteristic for solid closure) does not hold in the Fermat hypersurface domains

$$
R=K[X, Y, Z] /\left(X^{d}+Y^{d}+Z^{d}\right)
$$

for infinitely many degrees $d$ (Remark 4). In section 3 we apply the example to the $p$-adic setting of a preprint of C. Deninger and A. Werner (4]) and show that their category $\mathfrak{B}_{X_{0}}$ of vector bundles cannot contain all irreducible bundles of degree 0 .

I thank A. Langer and A. Werner for useful discussions.

\section{The EXAMPLE}

We now describe the example. Let $p$ denote a prime number and let $K$ denote a field of characteristic $p$. We consider the syzygy bundle $\operatorname{Syz}\left(X^{a}, Y^{a}, Z^{a}\right)(n), a \geq 1$, on $\mathbb{P}^{2}=\operatorname{Proj} K[X, Y, Z]$ given by the defining sequence

$$
0 \longrightarrow \operatorname{Syz}\left(X^{a}, Y^{a}, Z^{a}\right)(n) \longrightarrow \bigoplus_{3} \mathcal{O}(n-a) \stackrel{X^{a}, Y^{a}, Z^{a}}{\longrightarrow} \mathcal{O}(n) \longrightarrow 0 .
$$

For $a=1$ and $n=0$ this is just the Euler sequence for the cotangent bundle. The pull-back of this sequence under the $e$-th absolute Frobenius morphism $F^{e}: \mathbb{P}^{2} \longrightarrow$ $\mathbb{P}^{2}$ yields the sequences (setting $q=p^{e}$ )

$$
0 \longrightarrow\left(F^{e *} \operatorname{Syz}\left(X^{a}, Y^{a}, Z^{a}\right)\right)(m) \longrightarrow \bigoplus_{3} \mathcal{O}(m-a q) \stackrel{X^{a q}, Y^{a q}, Z^{a q}}{\longrightarrow} \mathcal{O}(m) \longrightarrow 0 .
$$

Therefore we have $\left(F^{e *} \operatorname{Syz}\left(X^{a}, Y^{a}, Z^{a}\right)\right)(m)=\operatorname{Syz}\left(X^{a q}, Y^{a q}, Z^{a q}\right)(m)$. For this bundle the following proposition holds.

Proposition 1. Let $K$ denote a field of positive characteristic $p$. Then the syzygy bundle $\operatorname{Syz}\left(X^{a}, Y^{a}, Z^{a}\right)$ on the projective plane $\mathbb{P}^{2}$ is strongly semistable, but it is not true that the restriction of this bundle is strongly semistable for all smooth curves of sufficiently high degree.

Proof. First note that the bundles $\operatorname{Syz}\left(X^{a q}, Y^{a q}, Z^{a q}\right)(m)$ do not have non-trivial global sections for $m<2 a q$ for all $a$ and $q$. So these bundles are semistable and also strongly semistable on the projective plane. For the main statement we have to show that for every number $d_{0} \in \mathbb{N}$ we find a degree $d \geq d_{0}$ and a smooth 
projective curve $C \subseteq \mathbb{P}^{2}$ of degree $d$ such that the restriction $\operatorname{Syz}\left(X^{a}, Y^{a}, Z^{a}\right) \mid C$ is not strongly semistable anymore.

Let $d_{0}$ be given and let $e$ be such that $a p^{e-1} \geq d_{0}$. Choose $d$ such that

$$
a p^{e-1}<d<3 a p^{e-1} / 2
$$

and such that $d$ is no multiple of $p$. Then

$$
a q=a p^{e}<d p<3 a q / 2 .
$$

Set $k=d p-a q>0$. Consider the smooth curve $C$ given by the Fermat equation $X^{d}+Y^{d}+Z^{d}=0$. On the affine cone $K[X, Y, Z] /\left(X^{d}+Y^{d}+Z^{d}\right)$ over this curve we have

$$
0=\left(X^{d}+Y^{d}+Z^{d}\right)^{p}=X^{d p}+Y^{d p}+Z^{d p}=X^{k} X^{a q}+Y^{k} Y^{a q}+Z^{k} Z^{a q} .
$$

This means that on the curve $C$ the syzygy bundle $\operatorname{Syz}\left(X^{a q}, Y^{a q}, Z^{a q}\right)(m)$ has a global non-trivial section given by $\left(X^{k}, Y^{k}, Z^{k}\right)$ of total degree $m=k+a q=d p$. But the degree of this syzygy bundle is by the short exact sequence

$$
\operatorname{deg}\left(\operatorname{Syz}\left(X^{a q}, Y^{a q}, Z^{a q}\right)(m)\right)=(2 m-3 a q) d=(2 d p-3 a q) d<0
$$

because of the second condition in the choice of $d$. So this bundle on the curve has negative degree but it has a global section $\neq 0$. Hence it is not semistable and $\operatorname{Syz}\left(X^{a}, Y^{a}, Z^{a}\right) \mid C$ is not strongly semistable.

\section{Some REMARKS}

Remark 2. Let $a q=a p^{e}<d p<3 a q / 2$ and $k=d p-a q$ as in the proof of Proposition 1 The global section in the restricted syzygy bundle gives the following short exact sequence on the Fermat curve $C=V_{+}\left(X^{d}+Y^{d}+Z^{d}\right) \subset \mathbb{P}^{2}$ :

$$
0 \longrightarrow \mathcal{O}_{C} \longrightarrow \operatorname{Syz}\left(X^{a q}, Y^{a q}, Z^{a q}\right)(d p) \longrightarrow \mathcal{O}_{C}(2 d p-3 a q) \longrightarrow 0
$$

This is of course the Harder-Narasimhan filtration of $\operatorname{Syz}\left(X^{a q}, Y^{a q}, Z^{a q}\right)(d p)$, and the maximal slope of $F^{e *} \operatorname{Syz}\left(X^{a}, Y^{a}, Z^{a}\right)$ is 0 and its minimal slope is $(2 d p-3 a q) d$, since $d$ is the degree of $\mathcal{O}_{C}(1)$.

A measure for the deviation from strong semistability of a locally free sheaf $\mathcal{S}$ is given by the number

$$
\lim _{e \in \mathbb{N}} \frac{\mu_{\max }\left(F^{e *} \mathcal{S}\right)-\mu_{\min }\left(F^{e *} \mathcal{S}\right)}{p^{e}} .
$$

This number is independent of a twist with an invertible sheaf. Thus in our example this number is $\geq(0-(2 d p-3 a q)) d / q=(3 a q-2 d p) d / q$. If we set $d=a p^{e-1}+1$, then this number is

$$
\frac{d\left(3 a q-2 p\left(a p^{e-1}+1\right)\right)}{q}=\frac{d(a q-2 p)}{q} \geq \frac{a p^{e-1}(a q-2 p)}{q}=a^{2} p^{e-1}-2 a .
$$

So for every $a$ this deviation measure is arbitrarily large (but finite if we divide by $d)$.

Remark 3. As mentioned in the introduction, there exist restriction theorems due to Bogomolov and to A. Langer for semistable sheaves which imply that the restriction to every smooth hypersurface of sufficiently high degree is again semistable. Here the degree bound does not depend only on the variety and the rank of the bundle 
(as in the restriction theorem of Flenner), but also on the discriminant of the bundle. The example above shows that in positive characteristic there cannot exist a restriction theorem for semistability for all smooth hypersurfaces with a degree bound depending only on data which are invariant under the Frobenius (such as the rank and invariants of the basic variety). For otherwise the restriction of a strongly semistable sheaf to a smooth hypersurface would also be strongly semistable.

Remark 4. The example is motivated by the geometric interpretation of the theory of tight closure. We refer to [7] and [8] for the background of this theory. In [3, Theorem 8.4] we proved that the tight closure of the ideal $\left(X^{a}, Y^{a}, Z^{a}\right)$ in $R=K[X, Y, Z] /(F)$, where $F$ is homogeneous, is given by

$$
\left(X^{a}, Y^{a}, Z^{a}\right)^{*}=\left(X^{a}, Y^{a}, Z^{a}\right)+R_{\geq 3 a / 2}
$$

under the condition that the syzygy bundle $\operatorname{Syz}\left(X^{a}, Y^{a}, Z^{a}\right)$ is strongly semistable on the curve $C=\operatorname{Proj} R$. Since this syzygy bundle is semistable on the projective plane, it follows in characteristic zero that this numerical formula for tight closure (or rather solid closure) holds in $R=K[X, Y, Z] /(F)$ for every $F$ of sufficiently high degree by the restriction theorem of Bogomolov. In fact this holds for every $F$ that is smooth of degree $\operatorname{deg}(F)=d \geq 3 a-1$; see [2] Proposition 6.2].

Here we want to remark that this is not true in positive characteristic. Let $p$ be a prime number. We show that for $a=2 b$ even the formula does not hold for $\left(X^{a}, Y^{a}, Z^{a}\right)$ on $F=X^{d}+Y^{d}+Z^{d}$ for suitable but arbitrary high degree $d$; in fact we will show that $X^{b} Y^{b} Z^{b} \notin\left(X^{a}, Y^{a}, Z^{a}\right)^{*}$ on such a curve. An even degree has the (dis-)advantage that there exist homogeneous elements having the critical degree $3 a / 2$.

Suppose as before that $a q<d p<3 a q / 2$ and $k=d p-a q$. From the HarderNarasimhan filtration (see Remark 22) we get by tensoring with $\mathcal{O}(m-d p)$ the short exact sequence

$$
0 \longrightarrow \mathcal{O}_{C}(m-k-a q) \longrightarrow \operatorname{Syz}\left(X^{a q}, Y^{a q}, Z^{a q}\right)(m) \longrightarrow \mathcal{O}_{C}(m+k-2 a q) \longrightarrow 0 .
$$

For $4 a q-2 k>2 m \geq 3 a q$ the invertible sheaf on the right has negative degree but the degree in the middle is $\geq 0$. Let $f=X^{r} Y^{s} Z^{t}$ be a monomial of degree $r+s+t=m$. Such an element yields via the defining sequence for $\operatorname{Syz}\left(X^{a q}, Y^{a q}, Z^{a q}\right)$ the Čech cohomology class

$$
\left(\frac{X^{r} Y^{s} Z^{t}}{X^{a q}},-\frac{X^{r} Y^{s} Z^{t}}{Y^{a q}}, 0\right) \in H^{1}\left(C, \operatorname{Syz}\left(X^{a q}, Y^{a q}, Z^{a q}\right)(m)\right) .
$$

The surjection $\operatorname{Syz}\left(X^{a q}, Y^{a q}, Z^{a q}\right)(m) \longrightarrow \mathcal{O}_{C}(m+k-2 a q)$ maps this cohomology class to the class

$$
-\frac{X^{r} Y^{s} Z^{t} Z^{k}}{X^{a q} Y^{a q}} \in H^{1}\left(C, \mathcal{O}_{C}(m+k-2 a q)\right) ;
$$

see [2, Remark 4.8].

If the formula $(*)$ would hold, then $X^{r} Y^{s} Z^{t} \in\left(X^{a q}, Y^{a q}, Z^{a q}\right)^{*}$, since $m \geq$ $3 a q / 2$. If however the cohomology class $\frac{X^{r} Y^{s} Z^{t} Z^{k}}{X^{a q} Y^{a q}}$ is not in $0^{*}$, which means by definition that $X^{r} Y^{s} Z^{t} Z^{k} \notin\left(X^{a q}, Y^{a q}\right)^{*}$ in $R=K[X, Y, Z] /(F)$, then (the corresponding geometric torsor of this class is affine and this is then true for the torsor corresponding to the first class) also $X^{r} Y^{s} Z^{t} \notin\left(X^{a q}, Y^{a q}, Z^{a q}\right)^{*}$. 
Now let $a=2 b \geq 2, d$ and $k$ be as before and set $m=3 a q / 2=3 b q$. Set $r=s=t=b q$, so that we have to look at the cohomology class

$$
\frac{X^{b q} Y^{b q} Z^{b q} Z^{k}}{X^{a q} Y^{a q}}=\frac{Z^{b q} Z^{k}}{X^{b q} Y^{b q}} \in H^{1}\left(C, \mathcal{O}_{C}(k-b q)\right),
$$

and we have to look for examples such that $Z^{b q+k} \notin\left(X^{b q}, Y^{b q}\right)^{*}$.

We now fix the degree $d=a p^{e-1}+1$, so that $k=p$ and $d>p$. Set $u=\lceil p / 2\rceil$. Then for $p \geq 3$ and $e \geq 2$ we have

$$
u d=\lceil p / 2\rceil\left(2 b p^{e-1}+1\right)=b p^{e}+p / 2+1 / 2+b p^{e-1} \geq b q+p .
$$

So it is enough to show that $Z^{u d} \notin\left(X^{b q}, Y^{b q}\right)^{*}$. But this class is

$$
-\frac{Z^{u d}}{X^{b q} Y^{b q}}=\frac{\left(X^{d}+Y^{d}\right)^{u}}{X^{b q} Y^{b q}}=\frac{X^{u d}+u X^{(u-1) d} Y^{d}+\ldots+Y^{u d}}{X^{b q} Y^{b q}}
$$

and we consider this class in $H^{1}\left(\mathbb{P}^{1}, \mathcal{O}(u d-2 b q)\right), \mathbb{P}^{1}=$ Proj $K[X, Y]$. The extreme summands on the left and on the right vanish, but for $e \geq 2$ and $p \geq 5$ we obtained that $(u-1) d<b q$, so the second summand is $\neq 0$ ( $p$ does not divide $u$ ). Therefore this class is $\neq 0$ and hence $\notin 0^{*}$, since $K[X, Y]$ is $F$-regular. It follows that the cohomology class does not belong to $0^{*} \subseteq H^{1}\left(C, \mathcal{O}_{C}(k-b q)\right)$ and so $(X Y Z)^{b} \notin$ $\left(X^{2 b}, Y^{2 b}, Z^{2 b}\right)^{*}$ in $R=K[X, Y, Z] /(F)$.

Problem 5. How does the strong semistability of $\operatorname{Syz}\left(X^{2}, Y^{2}, Z^{2}\right)$ on $X^{d}+Y^{d}+$ $Z^{d}=0$ depend on the prime number $p$ and the degree $d$ ? In particular, for fixed degree $d$, decide whether the set of prime numbers such that this syzygy bundle is strongly semistable is finite, infinite or contains almost all prime numbers? This question is both a special instance of a problem of Miyaoka (see [13, Problem 5.4]) and of a problem of tight closure theory ([6, Question 13]).

\section{How BIG ARE $\mathfrak{B}_{X_{\mathbb{C}_{p}}}$ AND $\mathfrak{B}_{\mathfrak{X}_{\mathfrak{o}}}$ ?}

C. Deninger and A. Werner establish in [4] a $p$-adic analog of the well-known analytic correspondence between flat vector bundles over a compact Riemann surface $X$ and GL-representations of the geometric fundamental group $\pi_{1}(X, x)$. They construct a functor which associates to a certain category of vector bundles on a smooth projective $p$-adic curve $X_{\mathbb{C}_{p}}$ a continuous representation of the algebraic fundamental group in a vector space over $\mathbb{C}_{p}$. However it remains open how big this category of bundles is; in particular, it is unclear whether it contains all irreducible (or all stable) bundles of degree 0.

We describe briefly the setting of [4]. Let $K$ denote a finite extension of the $p$-adic number field $\mathbb{Q}_{p}$, and let $\bar{K}$ denote its algebraic closure. Let $\mathbb{C}_{p}$ denote the completion of $\bar{K}$ with respect to the p-adic valuation. Let $X$ denote a smooth projective curve over $K$ and set $\bar{X}=X \otimes_{K} \bar{K}$ and $X_{\mathbb{C}_{p}}=X \otimes_{K} \mathbb{C}_{p}$. Let $\overline{\mathfrak{X}}$ denote a finitely presented flat and proper model over the valuation domain $\mathfrak{o}_{\bar{K}} \subset \bar{K}$ so that $\bar{X}$ is the generic fiber. The special fiber is then a not necessarily irreducible projective curve over $\mathfrak{o}_{\bar{K}} / \mathfrak{m}=\overline{\mathbb{F}}_{p}$.

They work with a special category $\mathfrak{B}=\mathfrak{B}_{\mathfrak{X}_{\mathfrak{o}}}$ of vector bundles on $\mathfrak{X}_{\mathfrak{o}}=\overline{\mathfrak{X}} \otimes \mathfrak{o}$, where $\mathfrak{o}$ denotes the valuation ring in $\mathbb{C}_{p}$. A vector bundle $E$ on $\mathfrak{X}_{\mathfrak{o}}$ belongs to this category if for every $n \geq 1$ there is a (generic étale and proper) "covering" $\pi: \mathcal{Y} \rightarrow \overline{\mathfrak{X}}$ (with some further technical properties) such that $\pi^{*} E_{n}\left(E_{n}=E /\left(p^{n}\right)\right)$ is trivial on the thickened fiber $\mathcal{Y}_{n}=\mathcal{Y} /\left(p^{n}\right)$. 
For the bundles in this category they construct a functor $\rho$ from $\mathfrak{B}_{\mathfrak{X}_{\mathrm{o}}}$ to the continuous representations $\operatorname{Rep}_{\pi_{1}(\bar{X}, \bar{x})}(\mathfrak{o})$ of the algebraic fundamental group $\pi_{1}(\bar{X}, \bar{x})$ ([4, Theorem 14]). This functor then also yields a functor (4, Theorem 23])

$$
\rho: \mathfrak{B}_{X_{\mathbb{C}_{p}}} \longrightarrow \boldsymbol{R e p}_{\pi_{1}(\bar{X}, \bar{x})}\left(\mathbb{C}_{p}\right),
$$

where the category $\mathfrak{B}_{X_{\mathbb{C}_{p}}}$ consists of the vector bundles on $X_{\mathbb{C}_{p}}$ which arise by restriction from a bundle in $\mathfrak{B}_{\mathfrak{X}}$ for some model $\overline{\mathfrak{X}}$ of $\bar{X}$ ([4, Definition 19]). The category $\mathfrak{B}_{X_{\mathbb{C}_{p}}}$ contains all invertible sheaves of degree 0 ([4, Theorem 23c]) and it is hoped that it contains all irreducible bundles of degree 0 ([4, Question $7 \mathrm{~d}]$ ). Our example shows that this is definitely not true for $\mathfrak{B}_{\mathfrak{X}_{0}}$ !

Since the Fermat equation $U^{d}+Y^{d}+Z^{d}=0$ is defined over $\mathbb{Z}$ we may consider the corresponding projective curve over any ring. We denote this curve over $\overline{\mathbb{Q}}_{p}=\bar{K}$ by $\bar{X}$ and the relative curve over $\mathfrak{o}_{\bar{K}}$ by $\overline{\mathfrak{X}}$, so this is our $p$-adic model for $\bar{X}$. We consider the bundle $E=\operatorname{Syz}\left(U^{2}, Y^{2}, Z^{2}\right)(3)$ on $\mathfrak{X}_{0}$. The determinant of this bundle is just the structure sheaf. This bundle has degree 0 and is stable and irreducible on $\bar{X}$, on $X_{\mathbb{C}_{p}}$ and on the special fiber $\mathfrak{X}_{\mathfrak{o}} \otimes \mathfrak{o} / \mathfrak{m}$, since this holds over every field (of any characteristic) for $d$ large enough.

The condition in the definition of $\mathfrak{B}_{\mathfrak{X}_{\mathrm{o}}}$, namely that there exists $\pi: \mathcal{Y} \rightarrow \overline{\mathfrak{X}}$ such that $\pi^{*}\left(E_{n}\right)$ is trivial, implies in particular that the bundle over the special fiber trivializes in a finite extension. A result of Lange and Stuhler ([Satz 1.9][10]) however says that a vector bundle on a smooth projective curve over an algebraically closed field of positive characteristic must be strongly semistable if it admits such a finite trivialization. But due to Proposition 1 this syzygy bundle is not strongly semistable for some degree $d$. Therefore for suitable degree $d$ the bundle $E=$ $\operatorname{Syz}\left(U^{2}, Y^{2}, Z^{2}\right)(3)$ does not belong to $\mathfrak{B}_{\mathfrak{X}_{\mathfrak{o}}}$.

It is however possible that there exists another model $\mathfrak{X}^{\prime}$ and a vector bundle $E^{\prime} \in \mathfrak{B}_{\mathfrak{X}_{\circ}^{\prime}}$ such that $E_{\mathbb{C}_{p}}^{\prime} \cong E_{\mathbb{C}_{p}}$. Then $E_{\mathbb{C}_{p}} \in \mathfrak{B}_{X_{\mathbb{C}_{p}}}$ would hold.

\section{REFERENCES}

[1] F. A. Bogomolov. Stability of vector bundles on surfaces and curves. In Einstein metrics and Yang-Mills connections. Proceedings of the 27th Taniguchi international symposium 1990, volume 145 of Lect. Notes Pure Appl. Math., pages 35-49, Dekker, New York, 1993. MR.1215277(94i:14021)

[2] H. Brenner. Computing the tight closure in dimension two. To appear in Mathematics of Computation, 2005.

[3] H. Brenner. Slopes of vector bundles and applications to tight closure problems. Trans. Amer. Math. Soc., 356(1):371-392, 2004. MR2020037

[4] C. Deninger and A. Werner. Vector bundles and p-adic representations I. Preprint, ArXiv, 2003.

[5] H. Flenner. Restrictions of semistable bundles on projective varieties. Comment. Math. Helv., 59:635-650, 1984. MR0780080 (86m:14014)

[6] M. Hochster. Tight closure in equal characteristic, big Cohen-Macaulay algebras, and solid closure. Contemp. Math., 159:173-196, 1994. MR1266183 (95a:13012)

[7] C. Huneke. Tight Closure and Its Applications. AMS, 1996. MR.1377268 (96m:13001)

[8] C. Huneke. Tight closure, parameter ideals, and geometry. In Six Lectures on Commutative Algebra. Birkhäuser, 1998. MR:1648666(99j:13001)

[9] D. Huybrechts and M. Lehn. The Geometry of Moduli Spaces of Sheaves. Viehweg, 1997. MR:1450870 (98g:14012)

[10] H. Lange and U. Stuhler. Vektorbündel auf Kurven und Darstellungen der algebraischen Fundamentalgruppe. Math. Zeitschrift, 156:73-83, 1977. MR0472827 (57:12517)

[11] A. Langer. Semistable sheaves in positive characteristic. Ann. Math., 159:251-276, 2004. MR 2051393 
[12] V. B. Mehta and A. Ramanathan. Semistable sheaves on projective varieties and the restrictions to curves. Math. Ann., 258:213-226, 1982. MR0649194 (83f:14013)

[13] Y. Miyaoka. The Chern class and Kodaira dimension of a minimal variety. In Algebraic Geometry, Sendai 1985, volume 10 of Adv. Stud. Pure Math., pages 449-476, 1987. MR0946247 (89k:14022)

Department of Pure Mathematics, University of Sheffield, Hicks Building, HounsField Road, Sheffield S3 7RH, United Kingdom

E-mail address: H.Brenner@sheffield.ac.uk 